\title{
Emotional/Behavioural Problems and Functional Impairment in Children with Attention-Deficit/Hyperactivity Disorder
}

\author{
M Tengsujaritkul, O Louthrenoo, N Boonchooduang
}

\begin{abstract}
Objective: This study aimed to compare the emotional/behavioural problems and functional impairment between early school-age children with attention-deficit/hyperactivity disorder (ADHD) and non-ADHD controls. Factors associated with behavioural problem scores were also evaluated.

Methods: Children aged 6 to 10 years who were diagnosed with ADHD based on the Swanson, Nolan and Pelham version IV Scale (SNAP-IV) and the DSM-5 criteria for ADHD were compared with ageand sex-matched controls with negative SNAP-IV results in terms of emotional/behavioural problems (as assessed by the Child Behavioural Checklist) and functional impairment (as assessed by the Strength and Difficulties Questionnaire).

Results: 40 children with ADHD and 40 non-ADHD controls with a mean age of $8.40 \pm 1.44$ years were included. The ADHD group scored significantly higher than controls in terms of all eight subscales of emotional/behavioural problems $(\mathrm{p}<0.001$ to $\mathrm{p}<0.01)$. Regarding functional impairment, the ADHD group reported significantly higher scores than controls in terms of total difficulties, conduct problems, and hyperactivity (all $\mathrm{p}<0.01)$; and significantly lower prosocial scores $(\mathrm{p}<0.03)$. In the multiple linear regression analysis, among the ADHD group, comorbid medical disorders were associated with higher total problem score, internalising behaviour problems score, and externalising behaviour problems score; whereas combined subtype ADHD was associated with higher total difficulties score.

Conclusions: Early school-age children with ADHD have more emotional/behavioural problems and functional impairment than non-ADHD controls, and they need further evaluation and intervention for psychosocial functioning, particularly those with comorbid medical disorder or combined subtype ADHD.
\end{abstract}

Key words: Attention deficit disorder with hyperactivity; Child; Emotions; Problem behavior

\footnotetext{
M Tengsujaritkul, Department of Paediatrics, Faculty of Medicine, Chiang Mai University, Chiang Mai, Thailand

O Louthrenoo, Department of Paediatrics, Faculty of Medicine, Chiang Mai University, Chiang Mai, Thailand

$N$ Boonchooduang, Department of Paediatrics, Faculty of Medicine, Chiang Mai University, Chiang Mai, Thailand
}

Address for correspondence: $O$ Louthrenoo, Department of Paediatrics, Faculty of Medicine, Chiang Mai University, Chiang Mai, 50200, Thailand.

Email: orawan.l@cmu.ac.th

Submitted: 20 March 2019 Accepted: 9 January 2020

\section{Introduction}

Attention-deficit/hyperactivity disorder (ADHD) is a common childhood-onset neurodevelopmental disorder, affecting $3.4 \%$ to $5.3 \%$ of school-age children worldwide, ${ }^{1-3}$ and $5.1 \%$ to $8.1 \%$ of school-age children in Bangkok, Thailand. ${ }^{4-6}$ ADHD is often associated with school underachievement, social difficulties, and mental health problems. Because of its long-term psychosocial burden and impaired functioning, ADHD has a great impact on children, their families, school, and community. In addition,
ADHD symptoms may persist, and children with ADHD are at greater risks of adverse outcomes in adolescence and adulthood. ${ }^{7}$ Adults with ADHD are likely to have comorbidities of substance/alcohol use problems and forensic record, as well as functional impairment. ${ }^{8}$

The age when functional deficits emerge in children with ADHD is not known. Preschool age children diagnosed with ADHD are at risk for poorer functioning from early life and display more behavioural problems and have less social skills than controls. ${ }^{9}$ Early primary school children with ADHD have lower language and cognitive performance, ${ }^{10}$ as well as poorer academic performance and mental health. ${ }^{11}$ Some impairment such as social skills can be more obvious when children become older. ${ }^{12}$ Adolescents with ADHD exhibit more oppositional defiant disorder, anxiety/ depression, major functional impairment, and reduced quality of life than non-ADHD peers. ${ }^{13}$

The relationships between internalising/externalising behaviour problems and children with and without ADHD have been examined. Internalising behaviours reflect the emotional state of children including depressive problems, anxiety problems, or somatic complaints, whereas externalising behaviours describe aggressive and delinquent 
problems. Both community-based and clinic-based children with ADHD, aged 6 to 15 years, have significantly higher internalising and externalising behaviours scores than non-ADHD controls as reported by their parents. ${ }^{14}$ Internalising behaviours in children with ADHD are related to peer victimisation both concurrently and at a 6-month follow-up, and these children are at increased risk for peer victimisation. ${ }^{15}$ Children with $\mathrm{ADHD}$ and co-occurring internalising/externalising behaviour disorders are prone to health-related impairments. ${ }^{16}$ Children with ADHD and comorbid externalising behaviour disorder have poorer social skills than those with ADHD alone. ${ }^{17}$

There is limited information on functional impairment in young children with ADHD in Thailand. This study aimed to compare the emotional/behavioural problems and functional impairment between early school-age children with ADHD and non-ADHD controls. Factors associated with behavioural problem scores were also evaluated. It was hypothesised that early school-age children with ADHD would have more emotional/behavioural problems and poorer functioning across all domains.

\section{Methods}

This study was approved by the ethics committee of the Faculty of Medicine, Chiang Mai University (reference: PED-2560-04463). Written informed consents were obtained from all parents. Children aged 6 to 10 years with ADHD were enrolled from the Developmental-Behavioural Clinic at a tertiary care centre from September 2017 to August 2018 and were followed up for at least 1 year. It is challenging for diagnosing and managing ADHD at preschool age. ${ }^{18}$

The diagnosis of ADHD was made by developmental paediatricians using the Swanson, Nolan, and Pelham version IV Scale (SNAP-IV) and the Diagnostic and Statistical Manual of Mental Disorders, 5th edition (DSM-5). ${ }^{19}$ Comorbid medical disorders including overweight/obesity, allergic diseases, and others were evaluated by paediatric specialists. Age- and sex-matched children from the same hospital with negative SNAP-IV results were enrolled as controls who were neither clinically diagnosed with ADHD by physicians nor reported to have ADHD symptoms by parents. Moreover, the parent rating score of the SNAP-IV less than the cutoff was used to confirm parents' reports of no clinical diagnosis of ADHD.

Exclusion criteria were children who had cognitive impairment, severe medical conditions, did not go to school, or whose parents had insufficient Thai language to be interviewed or complete questionnaires. Children who had ADHD with comorbid autism spectrum disorder were also excluded.

Three measurement tools were used. The Thai version of the SNAP-IV ${ }^{20-22}$ has 26 items rated on a 4-point scale (0 to 3 ) by both parents and teachers involving 18 ADHD symptoms (nine for inattentive and nine for hyperactiveimpulsive) and eight oppositional defiant disorder symptoms.
The Thai version of the Child Behaviour Checklist ${ }^{23,24}$ is rated by parents to evaluate emotional/behavioural problems in those aged 6 to 18 years. It is a widely used tool to identify problem behaviours. It comprises 113 items in eight subscales including anxiety/depression, social withdrawal, somatic complaints, social problems, thought problems, attention problems, delinquent behaviours, and aggressive behaviours, comprising internalising, externalising, and total behavioural problem scores. Higher scores indicate more emotional and behavioural problems.

The Thai version of the Strengths and Difficulties Questionnaire ${ }^{25,26}$ is used to evaluate functional impairment of children by parents. It is a short behavioural screening questionnaire for 3 to 16 year old and has high levels of reliability and validity for assessing psychopathology in children and adolescents worldwide. ${ }^{27-29}$ It comprises 25 items in five subscales including emotional difficulties, conduct difficulties, hyperactivity, peer problems scores, and prosocial subscales. The total problem score is the sum of the first four subscales, and higher scores indicate a greater number of problems or difficulties. The prosocial behaviour subscale is evaluated separately, and higher scores indicate better prosocial behaviour.

The SPSS Statistics (version 22.0, IBM, Armonk [NY], USA) was used for data analysis. Sample size was calculated from a study of functional impairment in the ADHD and control groups. ${ }^{14}$ To achieve the power of 0.80 and $\alpha$ of 0.05 , the number of participants was estimated to be 40 for each group..$^{30,31}$ The ADHD and control groups were compared using the Chi-squared test for categorical variables or the Student's t-test for continuous variables. A $p$ value of $<0.05$ was considered statistically significant. Univariate analysis was used to assess the association between variables and the total scores of the Child Behaviour Checklist and the Strengths and Difficulties Questionnaire. Variables with a p value of $<0.25$ (age, sex, ADHD subtype, and comorbid medical disorders) were included in multiple linear regression analysis.

\section{Results}

40 children with ADHD and 40 non-ADHD controls with a mean age of $8.40 \pm 1.44$ years were included. In the ADHD group, male children and ADHD combined subtype were more common (Table 1). All children with ADHD received short-acting stimulants medication for 6 months to 2 years. Comorbid disorders with minor medical conditions were similar between the two groups.

The ADHD group scored significantly higher than controls in terms of all eight subscales of emotional/ behavioural problems in the Child Behaviour Checklist $(\mathrm{p}<0.001$ to $\mathrm{p}<0.01$, Table 2$)$. In the ADHD group, 15 and 18 children were rated to have clinical internalising and externalising behaviour problems, respectively, whereas in the control group, the corresponding numbers were 9 and 3 .

Regarding functional impairment measured by the Strengths and Difficulties Questionnaire, the ADHD group 
Table 1. Characteristics of children with attention-deficit/hyperactivity disorder (ADHD) and controls

\begin{tabular}{|lccc|}
\hline Variables & ADHD $(\mathbf{n}=\mathbf{4 0})^{*}$ & ${\text { Controls }(\mathbf{n}=\mathbf{4 0})^{*}}^{*}$ & p Value \\
No. $(\%)$ of male children & $33(82.5)$ & $33(82.5)$ & $>0.99$ \\
\hline Age, y & $8.46 \pm 1.53$ & $8.34 \pm 1.37$ & 0.72 \\
\hline ADHD subtypes & $7(17.5)$ & & - \\
Inattentive & $5(12.5)$ & - & - \\
Hyperactive-impulsive & $28(70.0)$ & - & - \\
Combined & $16(40.0)$ & $11(27.5)$ & 0.24 \\
\hline Comorbidity & $410.0)$ & $3(7.5)$ & \\
Overweight/obesity & $7(17.5)$ & $5(12.5)$ & 0.38 \\
Allergic problems & $5(12.5)$ & $3(7.5)$ & 0.77 \\
Others & $40.89 \pm 7.76$ & $39.36 \pm 6.90$ & 0.24 \\
\hline Father age, y & $38.53 \pm 7.53$ & $38.05 \pm 6.95$ & \\
\hline Mother age, y & $31(77.5)$ & $35(87.5)$ & $5(12.5)$ \\
\hline Parental marital status & $9(22.5)$ & & \\
Couple & Single or separate & & \\
\hline
\end{tabular}

Data are presented as mean \pm standard deviation or No. (\%)

Table 2. Emotional/behavioural problems and functional impairment in the attention-deficit/hyperactivity disorder (ADHD) and control groups

\begin{tabular}{|lcccc|}
\hline Measurement & ADHD $(\mathbf{n}=\mathbf{4 0})^{*}$ & Controls $(\mathbf{n}=\mathbf{4 0})^{*}$ & $\begin{array}{c}\text { Mean difference } \\
(\mathbf{9 5 \%} \text { CI) }\end{array}$ & p Value \\
Child Behaviour Checklist total score & $51.10 \pm 23.12$ & $22.58 \pm 19.75$ & $28.53(18.95-38.10)$ & $<0.001$ \\
Internalising behaviour problems & $10.00 \pm 6.98$ & $5.60 \pm 5.53$ & $4.40(1.60-7.20)$ & 0.003 \\
Anxious / depressed & $4.35 \pm 3.61$ & $2.48 \pm 2.50$ & $1.88(0.49-3.26)$ & 0.01 \\
Withdrawn & $2.70 \pm 2.24$ & $1.35 \pm 1.82$ & $1.35(0.44-2.26)$ & 0.004 \\
Somatic complaints & $2.95 \pm 2.51$ & $1.78 \pm 2.35$ & $1.18(0.09-2.26)$ & 0.03 \\
Externalising behaviour problems & $15.40 \pm 8.03$ & $5.65 \pm 6.42$ & $9.75(6.51-12.99)$ & $<0.001$ \\
Delinquent behaviour & $4.15 \pm 2.75$ & $1.53 \pm 1.72$ & $2.63(1.60-3.65)$ & $<0.001$ \\
Aggressive behaviour & $11.25 \pm 6.06$ & $4.13 \pm 4.84$ & $7.13(4.69-9.57)$ & $<0.001$ \\
Other problems & $7.23 \pm 3.68$ & $3.38 \pm 2.76$ & $3.85(2.40-5.30)$ & $<0.001$ \\
Social problems & $5.38 \pm 3.12$ & $2.43 \pm 2.55$ & $2.95(1.68-4.22)$ & $<0.001$ \\
Thought problems & $4.85 \pm 3.61$ & $2.03 \pm 2.19$ & $2.83(1.49-4.16)$ & $<0.001$ \\
Attention problems & $8.25 \pm 3.01$ & $3.50 \pm 3.37$ & $4.75(3.33-6.17)$ & $<0.001$ \\
\hline Strengths and Difficulties Questionnaire & & & & \\
Total difficulties score & $16.00 \pm 5.07$ & $10.95 \pm 5.68$ & $5.05(2.65-7.47)$ & $<0.001$ \\
Emotional symptoms & $2.75 \pm 2.20$ & $1.95 \pm 1.78$ & $0.80(-0.09-1.69)$ & 0.08 \\
Conduct problems & $3.48 \pm 1.57$ & $1.78 \pm 1.70$ & $1.70(0.97-2.43)$ & $<0.001$ \\
Hyperactivity & $6.30 \pm 1.83$ & $4.13 \pm 2.07$ & $2.18(1.31-3.04)$ & $<0.001$ \\
Peer problems & $3.48 \pm 1.65$ & $3.10 \pm 1.93$ & $0.38(-0.42-1.17)$ & 0.35 \\
Prosocial behaviour & $6.53 \pm 1.95$ & $7.50 \pm 2.00$ & $-0.98(-1.85$ to -0.10$)$ & 0.03 \\
\hline
\end{tabular}

Data are presented as mean \pm standard deviation

reported significantly higher scores than controls in terms of total difficulties, conduct problems, and hyperactivity (all $\mathrm{p}<0.01)$; and significantly lower prosocial scores $(\mathrm{p}<0.03)$ [Table 2].
In the multiple linear regression analysis, among the ADHD group, comorbid medical disorders were associated with higher total problem score, internalising behaviour problems score, and externalising behaviour problems 
Table 3. Factors associated with emotional/behavioural problems and functional impairment in children with attentiondeficit/hyperactivity disorder (ADHD)

\begin{tabular}{|c|c|c|c|c|}
\hline \multirow[t]{2}{*}{ Test } & \multicolumn{4}{|c|}{ Variable $^{*}$} \\
\hline & Age & Male sex & Combined subtype ADHD & Comorbidity \\
\hline \multicolumn{5}{|l|}{ Univariate analysis } \\
\hline \multicolumn{5}{|l|}{ Child Behaviour Checklist } \\
\hline Total problem score & 0.47 & 0.56 & 0.25 & 0.001 \\
\hline Internalising problems & 0.33 & 0.64 & 1.00 & $<0.001$ \\
\hline Externalising problems & 0.33 & 0.17 & 0.08 & 0.06 \\
\hline \multicolumn{5}{|l|}{ Strengths and Difficulties } \\
\hline \multicolumn{5}{|l|}{ Questionnaire } \\
\hline Total difficulties score & 0.77 & 0.52 & 0.01 & 0.10 \\
\hline \multicolumn{5}{|l|}{ Multiple linear regression } \\
\hline \multicolumn{5}{|l|}{ Child Behaviour Checklist } \\
\hline Total problem score & - & - & $9.39(-4.65-23.43), p=0.18$ & $23.47(10.34-36.60), p=0.001$ \\
\hline Internalising problems & - & - & - & 7.60 (3.71-11.49), $p<0.001$ \\
\hline Externalising problems & - & $\begin{array}{c}-2.74(-8.06-2.59) \\
\mathrm{p}=0.30\end{array}$ & $0.81(-3.58-5.20), \mathrm{p}=0.71$ & $7.85(3.88-11.82), p<0.001$ \\
\hline \multicolumn{5}{|l|}{ Strengths and Difficulties } \\
\hline \multicolumn{5}{|l|}{ Questionnaire } \\
\hline Total difficulties score & - & - & $4.47(1.29-7.65), p=0.007$ & $2.80(-0.17-5.78), p=0.06$ \\
\hline
\end{tabular}

Data are presented as $p$ value or unstandardised regression coefficients (95\% confidence interval), $p$ value

score of the Child Behaviour Checklist; whereas combined subtype ADHD was associated with higher total difficulties score of the Strengths and Difficulties Questionnaire (Table 3).

\section{Discussion}

In the present study, early school-age children with ADHD displayed significantly more emotional/behavioural problems and functional impairment than non-ADHD controls, despite receiving short-acting stimulants and behavioural management for 6 months to 2 years. This finding is consistent with that reported in other studies of children with ADHD aged 6 to 15 years, ${ }^{14} 6$ to 8 years, ${ }^{11}$ and 9 to 11 years. ${ }^{10}$

The ADHD group had significantly higher total difficulties score and lower prosocial behaviour performance than controls, as well as not significantly higher peer problems score. However, one study reported a significantly higher peer problems score in children with ADHD than controls in a community sample. ${ }^{11}$ The differences may be due to the fact that both parent and teacher reports were assessed in the previous study, but only the parent report was used in the present study. Peer problems could be viewed differently in different settings, so parents may perceive less peer problems. Children with combined subtype ADHD have more impaired social skills and are less liked by their peers than children with the inattentive or hyperactiveimpulsive subtype. ${ }^{32}$
In the present study, comorbid medical disorders in children with ADHD were associated with higher total behavioural problem scores, and the combined subtype ADHD was associated with higher total difficulties scores. This is consistent with a study reporting that children with combined subtype ADHD have more functional impairment than those with the inattentive subtype ADHD. ${ }^{33}$ In addition, there was no sex difference in the functional status of children with ADHD, probably because of the small number of girls in the ADHD group. Similarly, girls and boys with ADHD did not differ in terms of internalising or externalising behaviours in a community sample ${ }^{11}$ and a clinic-based sample. ${ }^{34}$

There are limitations to the present study. Participants were from a tertiary hospital so the findings may not be generalised in the community. The sample size was too small to evaluate factors associated with emotional/behavioural problems and functional impairment in the ADHD group, although the sample size was considered adequate based on a previous study. Information was obtained from parents only; reports from other sources such as teachers would give more information. Details of management including duration of treatment or age at diagnosis were not analysed, a longitudinal study with a larger sample size is warranted.

\section{Conclusion}

Early school-age children with ADHD have more emotional/behavioural problems and functional impairment 
than non-ADHD controls, and they need further evaluation and intervention for psychosocial functioning, particularly those with comorbid medical disorder or combined subtype ADHD.

\section{Acknowledgements}

The authors would like to thank the participated children and their families.

\section{Declaration}

The authors have no conflict of interest to disclose.

\section{References}

1. Polanczyk GV, Salum GA, Sugaya LS, Caye A, Rohde LA. Annual research review: a meta-analysis of the worldwide prevalence of mental disorders in children and adolescents. J Child Psychol Psychiatry 2015;56:345-65. Crossref

2. Polanczyk G, de Lima MS, Horta BL, Biederman J, Rohde LA. The worldwide prevalence of ADHD: a systematic review and metaregression analysis. Am J Psychiatry 2007;164:942-8. Crossref

3. Willcutt EG. The prevalence of DSM-IV attention-deficit/hyperactivity disorder: a meta-analytic review. Neurotherapeutics 2012;9:4909. Crossref

4. Wacharasindhu A, Panyyayong B. Psychiatric disorders in Thai school-aged children: I Prevalence. J Med Assoc Thai 2002;85(Suppl 1):S125-36

5. Benjasuwantep B, Ruangdaraganon N, Visudhiphan P. Prevalence and clinical characteristics of attention deficit hyperactivity disorder among primary school students in Bangkok. J Med Assoc Thai 2002;85(Suppl 4):S1232-40.

6. Visanuyothin T, Pavasuthipaisit C, Wachiradilok P, Arunruang P, Buranasuksakul $\mathrm{T}$. The prevalence of attention deficit/hyperactivity disorder in Thailand. J Ment Health Thai 2013;21:66-75.

7. Thapar A, Cooper M. Attention deficit hyperactivity disorder. Lancet 2016;387:1240-50. Crossref

8. Leung VM, Chan LF. A cross-sectional cohort study of prevalence, comorbidities, and correlates of attention-deficit hyperactivity disorder among adult patients admitted to the Li Ka Shing Psychiatric Outpatient Clinic, Hong Kong. East Asian Arch Psychiatry 2017;27:63-70.

9. DuPaul GJ, McGoey KE, Eckert TL, VanBrakle J. Preschool children with attention-deficit/hyperactivity disorder: impairments in behavioral, social, and school functioning. J Am Acad Child Adolesc Psychiatry 2001;40:508-15. Crossref

10. Loe IM, Balestrino MD, Phelps RA, Kurs-Lasky M, Chaves-Gnecco D, Paradise JL, et al. Early histories of school-aged children with attentiondeficit/hyperactivity disorder. Child Dev 2008;79:1853-68. Crossref

11. Efron D, Sciberras E, Anderson V, Hazell P, Ukoumunne OC, Jongeling $\mathrm{B}$, et al. Functional status in children with ADHD at age 6-8: a controlled community study. Pediatrics 2014;134:e992-e1000. Crossref

12. Sacchetti GM, Lefler EK.ADHD symptomology and social functioning in college students. J Atten Disord 2017;21:1009-19. Crossref

13. Bussing R, Mason DM, Bell L, Porter P, Garvan C. Adolescent outcomes of childhood attention-deficit/hyperactivity disorder in a diverse community sample. J Am Acad Child Adolesc Psychiatry 2010;49:595-605. Crossref

14. Gau SS, Lin YJ, Shang CY, Liu SK, Chiu YN, Soong WT. Emotional/ behavioral problems and functional impairment in clinic- and community-based children with attention-deficit/hyperactivity disorder in Taiwan. J Abnorm Child Psychol 2010;38:521-32. Crossref

15. Fogleman ND, Leaberry KD, Rosen PJ, Walerius DM, Slaughter KE.
Relation between internalizing behaviors, externalizing behaviors, and peer victimization among children with and without ADHD. Atten Defic Hyperact Disord 2018;10:209-22. Crossref

16. Sciberras E, Bisset M, Hazell P, Nicholson JM, Anderson V, Lycett K, et al. Health-related impairments in young children with ADHD: a community-based study. Child Care Health Dev 2016;42:70917. Crossref

17. Booster GD, Dupaul GJ, Eiraldi R, Power TJ. Functional impairments in children with ADHD: unique effects of age and comorbid status. J Atten Disord 2012;16:179-89. Crossref

18. Wolraich ML. It takes a full-service village to treat children with ADHD. Pediatrics 2016;138:e20162928. Crossref

19. Diagnostic and Statistical Manual of Mental Disorders, 5th Edition. Washington, DC: American Psychiatric Association; 2013.

20. Swanson JM, Kraemer HC, Hinshaw SP, Arnold LE, Conners CK, Abikoff $\mathrm{HB}$, et al. Clinical relevance of the primary findings of the MTA: success rates based on severity of ADHD and ODD symptoms at the end of treatment. J Am Acad Child Adolesc Psychiatry 2001;40:168-79. Crossref

21. Swanson J, Lerner M, March J, Gresham FM. Assessment and intervention for attention-deficit/hyperactivity disorder in the schools. Lessons from the MTA study. Pediatr Clin North Am 1999;46:9931009. Crossref

22. Pityaratstian N, Booranasuksakul T, Juengsiragulwit D, Benyakorn S. ADHD screening properties of the Thai version of Swanson, Nolan, and Pelham IV Scale (SNAP-IV) and Strengths and Difficulties Questionnaire (SDQ). J Psychiatr Assoc Thailand 2014;59:97-110.

23. Achenbach TM, Rescorla LA. Manual for the ASEBA preschool form \& profiles. Burlington, VT: University of Vermont, Research Center for Children, Youth, \& Families; 2000.

24. Achenbach TM, Ruffle TM. The Child Behavior Checklist and related forms for assessing behavioral/emotional problems and competencies. Pediatr Rev 2000;21:265-71. Crossref

25. Wongpiromsarn $\mathrm{Y}$, Wipulakorn $\mathrm{P}$, Nuanmanee $\mathrm{S}$, Woerner $\mathrm{W}$, Mongkol A. Strengths and Difficulties Questionnaire (SDQ) Thai improved version: change and administration. J Ment Health Thai 2011;19:128-34.

26. Woerner W, Nuanmanee S, Becker A, Wongpiromsarn Y, Mongkol A. Normative data and psychometric properties of the Thai version of the Strengths and Difficulties Questionnaire (SDQ). J Ment Health Thai 2011;19:42-57.

27. Goodman R. Psychometric properties of the strengths and difficulties questionnaire. J Am Acad Child Adolesc Psychiatry 2001;40:133745. Crossref

28. Theunissen MHC, de Wolff MS, Reijneveld SA. The Strengths and Difficulties Questionnaire self-report: a valid instrument for the identification of emotional and behavioral problems. Acad Pediatr 2019;19:471-6. Crossref

29. Salayev KA, Sanne B, Salayev R. Psychiatric and behavioural problems in children and adolescents with epilepsy. East Asian Arch Psychiatry 2017;27:106-14.

30. Bernard R. Fundamentals of Biostatistics. 5th ed. Duxbery: Thomson learning; 2000.

31. Ngamjarus C, Chongsuvivatwong V.n4Studies: Sample size and power calculations for android. The Royal Golden Jubilee PhD Program. The Thailand Research Fund \& Prince of Songkla University. 2014.

32. Willcutt EG, Nigg JT, Pennington BF, Solanto MV, Rohde LA, Tannock $\mathrm{R}$, et al. Validity of DSM-IV attention deficit/hyperactivity disorder symptom dimensions and subtypes. J Abnorm Psychol 2012;121:9911010. Crossref

33. Weiss M, Worling D, Wasdell M. A chart review study of the inattentive and combined types of ADHD. J Atten Disord 2003;7:1-9. Crossref

34. Sciberras E, Efron D, Schilpzand EJ, Anderson V, Jongeling B, Hazell P, et al. The Children's Attention Project: a community-based longitudinal study of children with ADHD and non-ADHD controls. BMC Psychiatry 2013;13:18. Crossref 